Les universités en quête de prestige dans le grand jeu de la concurrence : le rôle de la communication marketing et l'impact des palmarès

Lucia Granget

(2) OpenEdition

Journals

Édition électronique

URL : http://journals.openedition.org/communicationorganisation/793

DOI : 10.4000/communicationorganisation.793

ISSN : $1775-3546$

Éditeur

Presses universitaires de Bordeaux

Édition imprimée

Date de publication : 1 juin 2009

Pagination : 148-157

ISSN : $1168-5549$

Référence électronique

Lucia Granget, «Les universités en quête de prestige dans le grand jeu de la concurrence : le rôle de la communication marketing et l'impact des palmarès », Communication et organisation [En ligne],

35 | 2009, mis en ligne le 01 décembre 2012, consulté le 30 avril 2019. URL : http://

journals.openedition.org/communicationorganisation/793; DOI : 10.4000/

communicationorganisation.793 
Dossier : Repenser la communication des organisations publiques

\section{Résumé}

Le prestige d'une université tend à dépendre de sa capacité «à faire spectacle» et à organiser sa mise en scène nationale et internationale. Sur fond de comparaisons, de statistiques, de discours officiels alarmistes et de réforme, cet article est consacré aux stratégies de communication mises en œuvre par les universités alors que la réputation d'excellence d'un établissement semble désormais reposer sur des places occupées dans des classements fortement médiatisés.

\section{Mots-clés}

Classement, communication marketing, communication publique, marketing social, université.

\section{Abstract \\ The renown of a university tends to depend on its ability «to do a show» and organise its national and international staging. In a background of comparisons, statistic and official disturbing speeches, this article is devoted to the communication strategies implemented by universities while the reputation of excellence of an intitution, now seems to rest on the place in the highly hyped ranking.}

\section{Key-Words}

Marketing communication, publique communication, ranking, social marketing, university.

Lucia Granget est Maître de Conférences à l'Université du Sud Toulon-Var et chercheur au laboratoire I3M. Elle est responsable du master «e-rédactionnel». Son activité de recherche est centrée sur la communication des organisations avec notamment l'étude des pratiques et des enjeux de la communication dans les universités. 


\title{
Les universités en quête de prestige dans le grand jeu de la concurrence : le rôle de la communication marketing et l'impact des palmarès
}

\author{
Lucia Granget
}

granget@univ-tln.fr

L'université qui hier encore échappait à la logique instrumentale s'est engagée dans un système concurrentiel. Depuis 1945, "tous les modes d'organisation ont connu une diversification interne croissante, une ouverture disciplinaire maximale, une diversification des modèles pédagogiques en fonction de nouvelles clientèles sociales et une complexification administrative des modes de gouvernement des institutions, génératrices de tensions nouvelles entre les diverses parties prenantes »(Charle, Verger, 1994). Aujourd'hui, le terme d'excellence est désormais au cœur de tous les discours et pour atteindre cet idéal les établissements universitaires sont appelés à se dépasser. Le prestige d'une université tend à dépendre de sa capacité «à faire spectacle» et à organiser sa mise en scène nationale et internationale. C'est au rôle de la communication marketing dans un contexte de réforme et de logique de benchmarking que nous nous attachons dans la première partie de cet article. La seconde est consacrée à l'impact de la médiatisation des classements dans l'enseignement supérieur. Cette réflexion combine plusieurs approches. Une analyse du contenu des documents de communication des universités depuis 1998 a permis de révéler une amplification d'un discours emprunté à l'entreprise. L'étude de rapports et de discours officiels a mis en évidence l'exploitation d'un langage issu du management. La démarche est complétée par l'examen de l'impact sur la communication, des palmarès nationaux et internationaux dont les méthodologies basées sur des indicateurs quantitatifs posent des problèmes de choix, de construction et d'analyse.

La communication marketing en question: s'adapter ou se conformer?

\section{Vers le marketing social}

«Le marketing est devenu la discipline reine ou le modèle universel pour la communication sociale et cela bien au-delà de la seule activité publicitaire» (Floris, 2001). Dès les années 1960, les universitaires critiques nord américains se prononcent en faveur de l'adoption d'une 
démarche de type marketing public. Dans les années 1980, ils orientent leurs raisonnements vers la prise en considération des besoins de la population, la valorisation de l'image de service public et le renforcement de la légitimité des organisations publiques. Face aux représentations souvent négatives de ce secteur, les actions sont principalement orientées vers l'amélioration de la perception de la qualité des prestations et de l'efficacité des services rendus. Parallèlement, les entreprises ont orienté leurs thématiques de communication institutionnelle vers des valeurs véhiculées traditionnellement par le secteur public : l'éthique, le respect, la citoyenneté, la responsabilité sociale et sociétale, l'environnement. Pour Nicole D'Almeida (2001), la société devient une «affaire d'entreprise ", après que l'entreprise soit devenue une "affaire de société ». A partir des années 70, le terme de «marketing social» commence à être employé $\left(1^{\text {er }}\right.$ Congrès de Marketing Social, Bruxelles, 1975). «Le marketing social se définit comme l'application de la méthode marketing aux problèmes sociaux, aux organisations qui ont le souci de leur insertion dans la société et de leurs rapports avec les groupes et les publics de leur environnement, aux services publics et aux collectivités» (Serraf, 1985). Par la suite, Philip Kotler et Bernard Dubois (2001) proposent l'expression d' «optique du marketing sociétal». Le sociétal "soutient que le rôle d'une entreprise est de déterminer et de satisfaire les besoins, désirs et intérêts du marché-cible plus efficacement que la concurrence mais de le faire d'une manière qui améliore le bien-être du consommateur et de la société dans son ensemble ».

Les deux conceptions «sociales » et «sociétales» ont favorisé des approches plus globalisantes qui permettent d'introduire des perspectives utopiques de recherche d'une plus grande humanité des conditions de vie socio-économiques. Elles ont favorisé l'introduction du marketing à toutes les formes d'organisations. Plus l'organisation publique se trouve en situation concurrentielle, plus elle est contrainte de poursuivre des objectifs de conquête de parts de marché qui imposent la nécessité de valoriser une image et de faire savoir une légitimité.

Pourtant, la généralisation excessive des méthodes issues du marketing, peut comporter des risques et susciter des questions de fond avec principalement celles de l'égalité d'accès et de service public universel. Récemment, de nombreux auteurs (Giroux, 2006 ; Oblin, Vassort, 2006 ; Jourde, 2007 ; Abensour, 2007 ; ...) se sont interrogés sur les soumissions idéologiques et économiques des universités au monde de l'entreprise. 


\section{Des universités en quête de reconnaissance}

Les universités, depuis une vingtaine d'années, ont mis en place une palette de moyens et d'outils destinés à «stimuler» le public. C'est l'un des pièges traditionnels de la communication publique qui consiste à se limiter à la dimension technique en «créant des supports et des actions d'information tout en négligeant la question des choix politiques en amont et des conditions de réussite de ces choix» (Bartoli, 1997). Mais la spécificité des missions, des objectifs, des modes organisationnels et les principes structurants du système universitaire ont freiné l'adoption d'une approche agressive de la communication marketing. "Dans le champ du public, l'importance de la relation, fait que la communication ne saurait se satisfaire des principes d'information de masse concurrentielle ou du marketing qui visent à modifier l'offre en vue du partage entre compétiteurs » (Zémor, 2005). Mais la démarche orientée uniquement vers la diffusion de l'information nécessaire à l'activité de formation et de recherche se révèle aujourd'hui inadaptée dans une logique d'autonomie des universités et de système concurrentiel. Les universités aspirent à devenir des marques (dépôts de la Sorbonne Abu Dhabi, Paris IV, et de Sorbonne Business School, Paris I). L'objectif est de favoriser la circulation de la marque et de renforcer la réputation de l'établissement. Certaines ont choisi de délaisser leur nom administratif au profit d'une appellation plus « commerciale » en référence à un lieu, à un auteur, à un scientifique, à une histoire. D'autres affichent deux noms : celui de l'établissement et celui de leur PRES d'appartenance. Ces actions s'inscrivent dans une perspective de renforcement de la visibilité à l'international, prescrites dans les discours politiques, institutionnels et médiatiques. Il en est de même pour les messages véhiculés par les universités. Ils évoquent «l'excellence », " la qualité », « la performance », «l'efficacité », «l'innovation », « l'ouverture », « l'adéquation de l'offre »... Sous l'effet de la «Déclaration de Bologne», de la publicisation des classements internationaux, de la généralisation des outils de référencements technologiques et de la loi LRU, la rhétorique de l'excellence a envahi les discours de la communication institutionnelle des universités. Elle inscrit les universités dans une logique de prescription. Le terme d' "excellence » est utilisé pour tout. Il permet la formulation d'exigences illimitées en occultant les problèmes de fond. Il ne désigne jamais l'activité, le travail et le métier de l'enseignement et de la recherche. L'excellence valorise le monde universitaire et sa culture professionnelle alors qu'elle s'accompagne d'un ensemble de prescriptions managériales. 
Dossier : Repenser la communication des organisations publiques

\section{Nouveau langage et démarche de benchmarking}

Alors que la réforme intervient sur fond de comparaisons internationales, de statistiques et de discours officiels alarmistes visant à mettre en évidence les retards de l'Europe et de la France en matière de formation et de recherche, les services communication des universités optent pour une rhétorique du progrès. Ainsi les discours alarmistes et les discours sur la réussite constituent, en quelque sorte, les facettes d'une même médaille. Les constats de déclin des discours officiels se métamorphosent en promesses communicationnelles pour les universités. Cette tendance a pour conséquence la duplication des thématiques de communication à l'ensemble des établissements. Cette reproduction semble peu propice à l'élaboration d'un positionnement marketing et à la création d'une image de marque fonction de l'identité. Elle conduit à l'uniformité. Or, l'identité "forge le climat interne et elle marque toute parole externe » (Zémor, 2005). Ce concept jugé très utile en marketing a fait l'objet de vives critiques (Lévi-Straus, 1977 ; Mucchielli, 1999 ; Laplantine, 1999 ...). Les politiques de communication menées par les universités visent à la création d'une identité de marque spécifique. Elles visent la différenciation. Mais elles se heurtent, en amont, à la difficulté de développer une offre réellement distincte tandis qu'en aval, les directives ministérielles et les injonctions aux changements sclérosent l'usage d'une expression créative. Le langage de la communication des universités devient caméléon. Il s'imprègne de l'actualité et se nourrit de toutes les formes de discours pour coller aux attentes de l'espace public. Le langage du management se révèle. Il marque un changement, une modification des contours de la figure de l'étudiant et de l'enseignant. Le sens de la relation pédagogique se transforme. La notion de client et le concept de qualité se dévoilent. La logique de benchmarking, envisagée comme condition de l'amélioration des performances de la formation et de la recherche, se systématise, en brouillant la fonction essentielle de l'Université : élaborer et transmettre de nouveaux savoirs. Cette logique, qui vise à établir la mesure économique du progrès et à évaluer les innovations, s'appuie sur la définition d'un étalon de mesure benchmark. L'identification des meilleures pratiques permet de mettre en évidence les retards par rapport aux concurrents. La décision et l'action s'établissent sur une mesure du retard et des écarts par rapport à une normalisation de l'excellence. Il est alors nécessaire pour les décideurs de disposer d'indicateurs de mesure. Mais, le concept d'excellence peut-il être normalisé au moyen de quelques indicateurs ? Il tend à se matérialiser par les productions de classements qui favorisent l'identification d'une 
hiérarchie nationale et internationale. Depuis quelques années, les initiatives en la matière se sont multipliées. Il convient de s'interroger sur l'utilité de ces dispositifs pour émettre des jugements et éclairer l'action des politiques. "Toute analyse communicationnelle pour expliquer à la fois le fonctionnement et la genèse (ou la mort) des organisations nous ramène nécessairement au modèle ingénierique des échanges informationnels, modèle dans lequel l'individu est le vecteur absent de ces échanges »(Gramaccia, 2001).

\section{L'impact des classements : affichage ou analyse ?}

\section{Une prolifération de classements}

Le processus de Bologne, dont le but était de promouvoir la cohérence des cursus au niveau européen et la mobilité internationale, semble écarté au profit de celui de Lisbonne. Faire de l'Europe, "l'économie de la connaissance la plus compétitive », mobilise toutes les attentions. Aux objectifs du LMD se substituent ceux de la compétitivité, de la performance et de la réputation. À l'international, le système français accuserait un retard en matière de performance et de notoriété, amplement médiatisé et commenté dans les discours politiques et institutionnels, notamment à l'occasion de la sortie des résultats de classements internationaux. Ces classements relèvent-ils de l'information du public? Quelle est leur influence sur les décideurs? Apportent-ils un éclairage stratégique? Sont-ils la contrepartie de l'autonomie des universités? La multiplication des classements nationaux et internationaux selon la méthode du ranking contribue à faire progresser l'idée de concurrence entre établissements. Le ranking s'inscrit dans une logique américaine élitiste. A partir de 1980, certains journaux tels que Business Week, Forbes, Wall Street Journal, US News and World Report..., hiérarchisent les établissements et principalement les business schools. Les journaux anglais (Financial Times, The Economist), allemand (Zeit), canadien (Maclean's), italien (La Republica), espagnol (Excelencia), imitent cette initiative. Pour accroître leur audience et développer de nouveaux marchés publicitaires, certains supports de la presse nationale française se lancent également dans la publication régulière de palmarès (Le Point, l'Expansion, le Nouvel Economiste, l'Express, le Figaro, Courrier cadres, Challenges, Capital, Management, le Nouvel Observateur, Vie universitaire, l'Etudiant...). Des suppléments des quotidiens régionaux sont également diffusés. Financés exclusivement par la publicité, ils sont censés permettre aux futurs bacheliers d'effectuer des choix en matière de poursuite d'études. Certains établissements, et notamment les écoles, ont 
développé des politiques précises pour remplir des questionnaires et pour créer des liens avec les agences de notation. Ils consacrent également des parts importantes de leur budget de communication à l'achat d'espaces publicitaires. Ces différentes actions ont pour but de gagner des places dans les palmarès. Le lecteur peut en conséquence être induit en erreur. La prolifération de suppléments et de classements présente le risque de constituer un élément d'opacité et d'inégalité supplémentaire.

Les incidences de la médiatisation des classements internationaux

À l'international, les classements d'universités sont moins nombreux. On note principalement celui de Shanghaï (Université de Jiao Tong, Institut de l'éducation supérieure), celui publié par le Times Higher Education Supplement, celui réalisé par l'Ecole des Mines et le Webometrics (mesure de la présence des établissements sur internet). Les critiques nombreuses sur les méthodologies et les critères retenus ne parviennent pas à occulter l'affluence de commentaires catastrophés sur la mauvaise position des institutions françaises par rapport à leurs homologues américaines. Au sein des établissements, des stratégies sont envisagées pour tenter d'améliorer les résultats obtenus. Elles portent sur : la valorisation du prestige et la renommée de l'établissement (classement britannique), la répartition des moyens financiers et humains au profit de certaines disciplines (sciences, économie et psychologie), le recrutement d'étudiants étrangers de certains pays, de Prix Nobel et de médailles Fields, le regroupement d'établissements (classement chinois), l'amélioration de l'insertion professionnelle des diplômés dans les grandes entreprises mondiales (Ecole des Mines) et la présence de publications sur Internet (Google Scholar). Mais c'est principalement, le classement de Shanghaï qui a connu un retentissement majeur dans les médias, auprès des autorités en charge de la recherche et des présidents d'universités. Dans sa lettre de mission du 5 juillet 2007 au ministre de l'Enseignement supérieur et de la Recherche, le président de la République fixe comme objectif d'avoir 2 établissements classés dans les 20 premiers mondiaux et 10 dans les 100 meilleurs. Une enquête menée par le sénateur Joël Bourdin (2008) montre "l'influence du classement de Shanghaï, puisque celui-ci est considéré par $71 \%$ des établissements comme un outil d'évaluation utile ». Dans les médias, la mise en spectacle porte alternativement sur la mauvaise position de la recherche française et sur la réussite de certaines universités classées parmi les 50, 100, 200 et 500 premières. En fait, la médiatisation du classement de Shanghaï a surtout permis de mettre clairement en évidence la mise en concurrence des universités au niveau mondial et 
d'affirmer leur rôle majeur en matière de recherche académique. Ainsi, Albert Fert (2008) dans le Monde relève que «la méthode employée fait s'évaporer la moitié de la notation attribuée à la recherche française ». Le CNRS et les autres organismes de recherche ne sont pas pris en considération. De plus, les revues en langue française et celles, spécialisées dans les disciplines des lettres, des sciences humaines et du droit ne sont pas compabilisées. En provoquant la consternation ce classement, dont l'objectif initial (2003) était de situer les universités chinoises par rapport à leur environnement mondial, a renforcé en France la volonté de mettre en place des stratégies nouvelles (fusions d'universités, constitution de PRES, franchises, programmes communs, établissements offshore...). Ces démarches ont pour ambition de permettre de gagner mécaniquement des places même si le véritable problème de la recherche française se situe probablement ailleurs.

\section{Hiérarchiser ou cartographier en Europe pour stimuler le changement}

La Commission européenne finance actuellement un projet pilote aux Pays-Bas (CWTS de l'université de Leiden) fondé sur des indicateurs bibliométriques. Elle soutient également en Allemagne le CHE Excellence Ranking (Centrum für Hochschulentwicklung) dont l'objectif est de mettre en évidence les forces et faiblesses de chaque établissement selon plusieurs indicateurs (factuels et d'opinion) et fonction des attentes de chacun. Sur ce principe, l'UE envisage d'effectuer des classements diversifiés à la commande (mapping) combinant plusieurs indicateurs communs. En France, la conférence des présidents d'universités est désormais favorable à un classement européen. Le rapport du Sénat (2008) «Enseignement supérieur : le défi des classements » dénonce "la subjectivité des indicateurs et l'absence de neutralité ». Mais il préconise paradoxalement la création d'un classement européen ainsi que la mise à disposition du public des résultats au plan national des prochaines vagues d'évaluations (2008-2012) des unités de recherche et des établissements. Selon ce rapport, "les classements promeuvent un certain modèle universitaire fondé sur la transparence, la concurrence et l'autonomie d'établissement disposant de moyens à la hauteur des ambitions qu'un pays industrialisé peut légitimement se donner en matière de recherche. En augmentant les moyens alloués à l'enseignement supérieur et en réformant son système universitaire, la France tend à s'aligner sur un modèle promu par les classements, qui ont ainsi un effet mobilisateur positif ». Les classements constitueraient-ils une alternative sérieuse pour stimuler le 
changement? Il s'agit de faire adopter par les universités françaises «la culture de résultat» et de les inciter à participer activement à la «bataille mondiale dans laquelle d'autres pays ont pris de l'avance sur nous» (entretien de Valérie Pécresse, le Figaro, 6 août 2008). L'association internationale des universités et l'OCDE (Dill, Soo, 2005) montrent que sur 202 institutions de 41 pays interrogés, $50 \%$ utilisent les résultats des classements pour leur publicité, $70 \%$ souhaitent figurer parmi les $10 \%$ meilleurs établissements au niveau national et $71 \%$ parmi les meilleurs au niveau international. La lecture des conclusions de l'enquête, montre un attachement significatif à une hiérarchie pourtant bien fragile. La réputation, qui en découle, s'établit sur une nature de l'excellence floue et sur des critères excluant les inégalités financières et culturelles entre les territoires. Ainsi, l'impact des classements peut présenter le risque de la mise en œuvre de stratégie éloignée de tout fondement éthique. Par ailleurs, le rapport «Citation statistics» (2008) de l'Union internationale de mathématiques met en évidence l'illusion des statistiques dans le domaine de la bibliométrie. Il souligne les tendances aux comportements de comptables provoqués par l'utilisation de critères d'évaluation. Nancy J. Adler et Anne-Will Harzing (2009) recommandent, pour leur part, un «moratoire» immédiat des « rankings institutionnels ».

\section{Conclusion}

La course aux premières places dans les palmarès, la diffusion de messages prometteurs, les changements de noms, l'adoption du langage de la performance participent à l'officialisation d'une autre vision du monde universitaire. La tendance est à l'imitation d'une forme caricaturale du modèle anglo-saxon. Or, celui-ci, dans le même temps, ambitionne d'évoluer. Lindsay Waters (2008), l'un des plus hauts responsables éditoriaux des Presses Universitaires d'Harvard, précise que l'université a adopté "la norme de l'entreprise américaine » avec une "exigence de productivité » qui conduit «à la publication de plus en plus de non-sens ». La situation est peu plaisante car "l'institution universitaire et le libre usage de l'intelligence s'opposent l'une à l'autre ». Sur la scène nationale et internationale, le classement fait spectacle. Il contribue à justifier la nécessité du renforcement des dispositifs d'évaluation en France pour une liberté et une responsabilité des universités très contrôlées. Il érige la performance en culture, la quantité en qualité et le conformisme en imagination. Son style démonstratif devient plus important que sa substance. 
Les universités en quête de prestige...

\section{Bibliographie}

ADLER J.N., HARZING A.W.K., "When knowledge wins : Transcendingthe sens and nonsense of academic rankings", Academy of Management Learning \& Education, vol. 8, n $1,2009$.

BARTOLI A., Le management dans les organisations publiques, Dunod, Paris, 1997.

BOURDIN J., Enseignement supérieur: les défis des classements. Rapport Sénat, Paris, 2008.

CHARLES C., VERGER J., Histoire des universités, PUF, Paris, 1994.

DILL D., SOO M. "Academic quality, league tables, and public policy: a cross-national analysis of university ranking systems", Higher Education, 2005.

GIROUX A., Le pacte faustien de l'université, Liber, Montréal, 2006.

GRAMACCIA G., Les actes de langage dans les organisations, L'Harmattan, Paris, 2001.

JOURDE P., Université : La grande illusion, éd. L'esprit des péninsules, Paris, 2007.

KOTLER P., DUBOIS B., Marketing management : analyse, planification, contrôle, Publi-Union, $3^{\circ}$ édition, 2001.

LAPLANTINE F., Je, nous et les autres, éd. Le Pommier, Paris, 1999.

LEVI-STRAUSS C., L'identité, Quadrige, PUF, Paris, 1977.

MUCCHIELLI A., L'identité, Que sais-je, PUF, Paris, 1999.

OCDE, L'impact des politiques économiques sur l'investissement en éducation supérieure, 2007, http://tinyurl.com/33b7z3.

OBLIN N. VASSORT P., La crise de l'université française, L'Harmattan, Paris, 2006.

RENAUT A., Que faire des universités ? Bayard, Paris, 2002.

SERRAF G., Dictionnaire méthodologique du marketing, éd. d'Organisation, coll. Adetem- Marketing demain, Paris, 1985.

Union Internationale de mathématiques, "Citation statistics", 2008.

ZEMOR P., La communication publique, PUF, Paris, 2005 